\title{
Evidencia basada en la práctica en psicoterapia: el reto en Latinoamérica
}

\section{Practice-based evidence in psychotherapy: the challenge in Latin America}

\author{
Adriana Trujillo 10 RCID Clara Paz \\ ${ }^{1}$ Universidad El Bosque, Colombia \\ ${ }^{2}$ Universidad de Las Américas, Ecuador
}

Fecha correspondencia:

Recibido: noviembre 14 de 2019.

Aceptado: junio 11 de 2020.

Forma de citar:

Trujillo, A., \& Paz, C. (2020). Evidencia

basada en la práctica en psicoterapia:

el reto en Latinoamérica. Rev. CES

Psico, 13(3), 1-14.

Open access

(c) Copyright

Licencia creative commons

Ética de publicaciones

Revisión por pares

Gestión por Open Journal System

DOl: http://dx.doi.org/10.21615/

cesp.13.3.1

ISSN: 2011-3080

Sobre los autores:

1. Doctora de Psicología Clínica y de la Salud. Docente-Investigadora Facultad de Psicología, Universidad El Bosque.

2. Doctora en Personalidad y Comportamiento. DocenteInvestigadora Escuela de Psicología, Universidad de Las Américas.

Comparte

\section{Resumen}

El artículo presenta una revisión teórica sobre el paradigma de la Práctica Basada en la Evidencia y sobre el de la Evidencia Basada en la Práctica en el ámbito de la psicoterapia; se profundiza especialmente en este último paradigma y su asociación con la investigación de la efectividad en psicoterapia. Posteriormente, se contextualiza el funcionamiento de este último paradigma en los sistemas de salud y en particular en la atención en salud mental, haciendo hincapié en su aplicabilidad en la evaluación rutinaria y su potencial para contribuir al campo de la investigación en psicoterapia. Finalmente, se presentan algunas conclusiones sobre cómo promover la investigación orientada a la Evidencia Basada en la Práctica en contextos psicoterapéuticos, a partir de Redes de Investigación en la Práctica en los países latinoamericanos.

Palabras clave: Práctica Basada en la Evidencia, Evidencia Basada en la Práctica, Medidas de Proceso y Resultados del Tratamiento, Resultados en Psicoterapia, Psicoterapia, Investigación en Psicoterapia, Salud Mental.

\section{Abstract}

This article presents a theoretical review of the Evidence-Based Practice and the Practice-Based Evidence in psychotherapy, the latter is explained in depth considering its association with the research of the effectiveness in psychotherapy. Subsequently, the functioning of this last paradigm in health systems, particularly in mental health care is contextualized, emphasizing its applicability in routine evaluation and its potential to contribute to the field of research in psychotherapy. Finally, some conclusions are presented on how to promote research based on the paradigm of Practice-Based Evidence in psychotherapeutic contexts, based on Practice Research Networks in Latin American countries.

Keywords: Evidence-Based Practice, Practice-Based Evidence, Treatment Process and Outcome Measures, Psychotherapeutic Outcomes, Psychotherapy, Psychotherapy Research, Mental Health. 
En el contexto latinoamericano, de la misma forma que ocurre en otras regiones, la Práctica Basada en la Evidencia (PBE) ha sido el paradigma dominante; el cual se adopta en la práctica habitual a través de las guías clínicas que promueven tratamientos específicos basados en la evidencia (Holmqvist, Philips, \& Barkham, 2015). Alternativamente, la presente revisión enfatiza en el paradigma de la Evidencia Basada en la Práctica (EBP)cn el propósito de promover una forma diferente de pensar la evidencia, replanteando la forma en que se puede utilizar la práctica clínica para aprender más sobre el cambio en psicoterapia y contribuir a una comprensión más amplia de lo que ocurre en el espacio terapéutico (Green, \& Latchford, 2012)

\section{Evidencia basada en la práctica: el reto de la evaluación rutinaria en psicoterapia en Latinoamérica}

En las últimas décadas, se han hecho esfuerzos importantes para solventar las distancias existentes entre la investigación y la práctica en contextos terapéuticos; este trabajo confluye en pro de la rigurosidad y la calidad en la implementación de diversas terapias psicológicas (Barkham \& Mellor-Clark, 2003). Bajo este panorama, el paradigma de la Práctica Basada en la Evidencia (en adelante PBE) ha sido especialmente reconocido por su aporte a la rigurosidad en la práctica psicológica; sin embargo, otras propuestas metodológicas han emergido dando respuesta a las limitaciones de este modelo, a las necesidades de los profesionales y a las dinámicas propias de los sistemas de salud, tal es el caso del paradigma de la Evidencia Basada en la Práctica (en adelante EBP) (Barkham \& Mellor-Clark. 2000; Margison et al., 2000).

En el contexto latinoamericano, de la misma forma que ocurre en otras regiones, la PBE ha sido el paradigma dominante; el cual se adopta en la práctica habitual a través de las guías clínicas que promueven tratamientos específicos basados en la evidencia ( $\underline{H o l m q}-$ vist, Philips, \& Barkham, 2015). Alternativamente, la presente revisión enfatiza en el paradigma de la EBP con el propósito de promover una forma diferente de pensar la evidencia, replanteando la forma en que se puede utilizar la práctica clínica para aprender más sobre el cambio en psicoterapia y contribuir a una comprensión más amplia de lo que ocurre en el espacio terapéutico (Green, \& Latchford, 2012).

No se pretende que uno de los modelos prevalezca sobre el otro, por el contrario, el objetivo de este planteamiento es que tanto profesionales como investigadores puedan reconocer la importancia de ambos paradigmas, ya que en conjunto proporcionarán una base de conocimientos más sólida de las terapias psicológicas. De hecho, Barkham y Mellor-Clark (2003) recalcan que la obtención de información desde ambos modelos es absolutamente necesaria para enriquecer la psicoterapia.

Para entender los paradigmas de la PBE y la EBP, es necesario hacer referencia a los modelos de Eficacia y Efectividad en investigación, que, aunque plantean puntos de vista diferentes, en conjunto pretenden identificar las características esenciales del funcionamiento diferencial de las técnicas y procedimientos psicológicos (Nathan, 2007). La diferencia fundamental es que mientras el objetivo principal de los estudios de investigación relativos a la eficacia es estudiar si un tratamiento particular funciona bajo condiciones controladas, en los estudios de efectividad se pretende estudiar la generalización, la factibilidad y la rentabilidad de los tratamientos cuya eficacia ha sido comprobada (Jacobson \& Christensen, 1996).

El Instituto Nacional de Salud Mental en Estados Unidos (National Advisory Mental Health Council [NAMHC], 1999) destacó la importancia de la investigación en torno a la eficacia y la efectividad de los tratamientos psicológicos, pero además incluyó los conceptos de investigación práctica e investigación en sistemas y servicios. La investigación práctica pretende conocer cómo y qué tratamientos o servicios se proporcionan a las personas dentro de los sistemas de salud; adicionalmente, evalúa cómo mejorar estos tratamientos o la prestación de servicios realizada. Por otro lado, la investigación en sistemas y servicios se enfoca en aquellas cuestiones organizativas, financieras y de políticas a gran escala. Según Barkham y Mellor-Clark (2003), bajo este último enfoque se incluye el costo de varias opciones de atención, las diferentes estrategias para la promoción y el acceso óptimo al servicio, el efecto de la legislación y las políticas públicas que regulan la organización y prestación de servicios, y el efecto que los cambios en un sistema tienen en la entrega de servicios. 
Pág 3

En el año 2014, Kazdin presentó una lista de 320 intervenciones psicosociales que cumplen con la condición de estar basadas en la evidencia. Esta cifra representa un amplio número de intervenciones eficaces en el ámbito de la psicología; sin embargo, Kazdin, (2014) reconoce diversas fuentes de error en los estudios de eficacia.
En este sentido, el presente artículo tiene como objetivo presentar una revisión teórica de ambos paradigmas: la PBE y EBP en el ámbito de la psicoterapia, y profundiza especialmente en este último paradigma y su asociación con la investigación de la efectividad en psicoterapia. También se presentan algunas conclusiones sobre los retos y las estrategias para promover el uso del paradigma de la EBP en psicoterapia en el contexto latinoamericano.

\section{El paradigma de la práctica basada en la evidencia}

El paradigma de la PBE comenzó a cobrar impulso en la década de los 90's, cuando se planteó la necesidad de realizar una práctica médica basada en la evidencia científica (Lilienfeld, Ritschel, Lynn, Cautin, \& Latzman, 2013). La PBE se sustenta en la utilización de tratamientos empíricamente validados, apoyados por ensayos clínicos controlados. Aunque no existe consenso respecto a los criterios que determinan que un tratamiento se clasifique en la categoría de basado en la evidencia, en general, se parte de la base que éste ha producido cambio en al menos un ensayo controlado aleatorizado y sus efectos han sido replicados (White, Koss, \& Kazdin, 2011).

De allí que este paradigma contribuya específicamente a la investigación sobre la eficacia de los tratamientos, la cual debe ajustarse a pautas rigurosas respecto al diseño, selección de las muestras, procedimientos de evaluación, implementación del tratamiento y análisis de datos; todo ello con el objetivo de maximizar la validez interna. El ensayo controlado aleatorio cumple con estas pautas, convirtiéndose en el diseño representativo de los estudios de eficacia (McMorrow \& Carr, 2003).

En este tipo de diseño la muestra corresponde a un grupo de casos homogéneos que cumplen con los criterios para un diagnóstico específico y que no presentan ninguna comorbilidad. Las condiciones o grupos de tratamiento y control son asignadas al azar. El tratamiento debe ser administrado por terapeutas entrenados según un manual específico para un determinado número de sesiones. Las evaluaciones que tengan lugar antes y después del tratamiento, y durante el seguimiento deben ser realizadas por un equipo de evaluadores que desconocen la ubicación de los casos en los grupos de tratamiento o control. Estos procesos de evaluación deben incluir procedimientos confiables y válidos que permitan valorar adecuadamente la presencia de síntomas asociados al trastorno objetivo. Posteriormente, durante el seguimiento, los casos deben clasificarse según si la mejoría ha sido clínicamente significativa o no; además de acompañarse con los resultados estadísticos sobre las diferencias intergrupales en las tasas de mejoría o puntuaciones medias, calculados con los estadísticos inferenciales apropiados. Finalmente, si tras la aplicación del tratamiento se evidencia una mejoría mayor a la comprobada en el grupo control, y este resultado se ha replicado al menos una vez, entonces se puede afirmar que la eficacia de dicho tratamiento cuenta con respaldo empírico (McMorrow \& Carr, 2003).

En el año 2014, Kazdin presentó una lista de 320 intervenciones psicosociales que cumplen con la condición de estar basadas en la evidencia. Esta cifra representa un amplio número de intervenciones eficaces en el ámbito de la psicología; sin embargo, Kazdin, (2014) reconoce diversas fuentes de error en los estudios de eficacia. Por ejemplo, en las condiciones de control y comparación de los ensayos clínicos aleatorios, ninguna de las fuentes de comparación (sin tratamiento o lista de espera, tratamiento placebo, tratamiento habitual u otra condición de tratamiento) se encuentra libre de limitaciones respecto al nivel de control y en especial respecto a las atribuciones que se puedan hacer con referencia a los mecanismos implicados en el cambio. Lo mismo ocurre cuando se analizan los criterios de significación estadística y de tamaño del efecto por sobre los criterios de significancia clínica. Además, la elección de medidas que se ajustan mejor a las hipótesis del estudio 
Martínez-Taboas (2014) señala que la mayor parte de las publicaciones sobre estudios de eficacia de la psicoterapia están basadas en poblaciones estadounidenses y europeas, situación por la cual otras etnias y culturas, especialmente de poblaciones asiáticas y latinas, no pueden utilizar esta información para sus prácticas terapéuticas. y la forma en que se reportan los resultados pueden estar sesgados por el cumplimiento de las hipótesis iniciales. La principal fortaleza de los estudios de eficacia es la validez interna, relacionada principalmente con la rigurosidad de la metodología empleada; sin embargo, la validez externa se ve limitada por su escasa aplicabilidad en la práctica real (Gérvas \& Peréz, 2008). Esta reflexión no pretende demeritar el camino recorrido por la PBE, trata más bien de aclarar las características de un tratamiento empíricamente validado (O'Donohue \& Ferguson, 2006).

En este punto, es importante hacer hincapié en las limitaciones propias del contexto latinoamericano respecto a la investigación y aplicabilidad de las psicoterapias con respaldo empírico. Una revisión de 101 publicaciones iberoamericanas sobre ensayos controlados aleatorios para poner a prueba intervenciones cognitivo-conductuales, encontró que 40 fueron desarrollados en Latinoamérica e identificó que sólo siete hacían referencia a aspectos de adaptación cultural de la intervención psicológica realizada (Preciado, 2012). Estos datos evidencian que, aunque se realizan estudios sobre la eficacia de tratamientos psicológicos creados en países desarrollados, pocos atienden a las características particulares de la población en la que se lleva a cabo la intervención. Por tanto, aunque las intervenciones psicológicas estudiadas se propongan como eficaces en este contexto según los indicadores de cambio que se hayan planteado, estos tratamientos no necesariamente podrán ser aplicados en la práctica cotidiana de la psicoterapia con los mismos resultados obtenidos en el ensayo clínico aleatorizado. Una dificultad adicional que se presenta en la mayoría de los países latinoamericanos es la escasa inversión en investigación en salud mental, lo cual repercute en el diseño, implementación, adaptación, validación y divulgación de estrategias de evaluación, formulación e intervención con apoyo empírico y que además se encuentren ajustadas a las necesidades específicas de cada población (Kazarian \& Evans, 2001).

Martínez-Taboas (2014) señala que la mayor parte de las publicaciones sobre estudios de eficacia de la psicoterapia están basadas en poblaciones estadounidenses y europeas, situación por la cual otras etnias y culturas, especialmente de poblaciones asiáticas y latinas, no pueden utilizar esta información para sus prácticas terapéuticas. Dada la influencia de los factores culturales, las limitaciones del paradigma de la PBE en lo que a la generalización de resultados se refiere, y los aspectos contextuales propios de los países de América Latina (Resnicow, Soler, Braithwaite, Ahluwalia, \& Butler, 2000; Zane, Hall, Sue, Young, \& Núñez, 2004), es necesario el desarrollo de estudios de eficacia de la psicoterapia que contemplen las variables propias de cada contexto.

\section{El paradigma de la evidencia basada en la práctica}

Barkham y Margison (2007) definen el paradigma de la EBP en psicoterapia como el uso minucioso, explícito y juicioso de la evidencia obtenida de los entornos de práctica clínica, lo que permite tomar decisiones sobre la atención de pacientes individuales. Mediante la EBP en psicoterapia se integra tanto la experiencia clínica individual como las características propias de prestación del servicio con la mejor evidencia disponible, la cual proviene de una rigurosa actividad de investigación llevada a cabo en entornos clínicos rutinarios. El paradigma de la EBP suele asociarse con la investigación sobre la efectividad en psicoterapia, tiene lugar en los servicios de salud en donde no suele ser factible la aplicación de criterios estrictos de exclusión, la aleatorización de condiciones de tratamiento y control, e incluso en muchos casos la utilización de tratamientos descritos en manuales de intervención (McMorrow \& Carr, 2003). Por lo cual, la EBP busca maximizar la validez externa, la cual se refiere al grado en el que las conclusiones sobre el tratamiento psicológico y sus resultados pueden ser generalizados a entornos diferentes en los cuales se han realizado los estudios de eficacia (Nathan, Stuart, \& Dolan, 2000). Para Barkham, Stiles, Lambert 
Desde el punto de vista de la Asociación de Psicología Americana (APA, 2006), la Evidencia Basada en la Práctica en psicoterapia indica la utilidad clínica de los tratamientos, que incluye aspectos como su aplicabilidad, viabilidad y utilidad en los entornos en los cuales se ofrecerán los servicios. y Mellor-Clark (2010), la EBP pretende capturar la realidad de la práctica cotidiana y encontrar formas de evaluarla y mejorarla adoptando un enfoque de abajo hacia arriba, es decir, comenzando con el trabajo de los profesionales y construyendo la base de evidencia hacia los principios y políticas de intervención, en lugar de arriba hacia abajo como ocurre en los estudios de eficacia.

Desde el paradigma de la EBP, la comparación de los grupos de tratamiento y control respecto a variables demográficas y de diagnóstico se realiza post-hoc, utilizando estadísticos inferenciales (un análisis de covarianza puede ser útil en estos casos, por ejemplo); y los profesionales ofrecen tratamientos apoyados por una amplia variedad de perspectivas teóricas, adaptándolos a las necesidades únicas de cada paciente, lo cual puede ser más apropiado y efectivo que un tratamiento psicológico manualizado (descrito operacionalmente) (McMorrow \& Carr, 2003).

Desde el punto de vista de la Asociación de Psicología Americana (APA, 2006), la EBP en psicoterapia indica la utilidad clínica de los tratamientos, que incluye aspectos como su aplicabilidad, viabilidad y utilidad en los entornos en los cuales se ofrecerán los servicios. Este enfoque abarca la investigación en aspectos relacionados con las características de los pacientes, los terapeutas, los entornos y la interacción entre estos factores; el funcionamiento de los tratamientos en varias modalidades de aplicación; la viabilidad con la que se pueden administrar tratamientos a pacientes en entornos reales y los costos asociados con la aplicación de las intervenciones. Un ejemplo de ello es el estudio realizado por Barkham, Delgadillo, Firth y Saxon (2018), que se enfoca en la variabilidad de los modelos de tratamiento psicológico según el tipo de pacientes, terapeutas y grupos, así como, el nivel de servicio, vecindarios y regiones.

La aplicación del paradigma de la EBP en psicoterapia se construye sobre una amplia recolección de datos, considerablemente mayor a la realizada bajo el paradigma de la PBE; lo cual solo es posible a través de Redes de Investigación en la Práctica (RIP) en psicoterapia, definidas en función de la colaboración entre profesionales mediante la recolección de información sobre lo que sucede en la práctica cotidiana (Audin et al., 2001). Los participantes de las RIP recogen datos de sus entornos de ejercicio profesional, uniendo las actividades de investigación y práctica con el objetivo de obtener información clínicamente significativa y científicamente rigurosa; de este modo, los profesionales en salud mental adquieren conciencia y se apropian de la investigación y de sus aportes en los diversos niveles de prestación del servicio (Barkham \& Mellor-Clark, 2003; Borkovec, Echemendia, Ragusea, \& Ruiz, 2001). Posteriormente, se profundizará sobre este modelo de trabajo de las RIP.

Si bien los estudios basados en la práctica cumplen una serie de características, estos rasgos no pueden ser considerados filtros rígidos, de la manera como ocurre en los estudios de eficacia, dado que, al enmarcarse en la práctica cotidiana, las limitaciones o criterios excluyentes sobre los datos que se pueden recolectar son prácticamente inexistentes. No obstante, Shadish et al. (1997) formularon una serie de criterios que definen a los estudios basados en el paradigma de la EBP en psicoterapia: a) ser realizado en centros comunitarios de salud mental o centros de atención primaria, b) involucrar clientes remitidos a través de rutas habituales, c) involucrar profesionales experimentados y profesionales con una carga de trabajo regular en lugar de terapeutas en capacitación, d) no utilizar manuales de tratamiento, e) no supervisar la implementación del tratamiento, e f) incluir participantes heterogéneos en cuanto a sus características personales y el tipo de problemas que presentan. 
Como cualquier otro paradigma, el de la Evidencia Basada en la Práctica no está exento de amenazas a su validez. La primera de ellas se relaciona con la calidad de los datos, aspecto relacionado con la adecuada elección de las medidas de evaluación y de la completitud de los datos.
Barkham et al. (2010) proponen un modelo que resume el proceso de investigación basada en la práctica en psicoterapia. En primer lugar, se identifica un sistema para la medición del proceso o del resultado, lo cual requiere definir las áreas de interés y la forma en que serán cuantificados los datos, y en consecuencia desarrollar o adoptar procesos de medición rutinaria utilizados de forma generalizada en el servicio. Definitivamente, en este enfoque, el uso de un sistema de medición precede a la identificación de cualquier pregunta; lo cual difiere de la metodología tradicional de los ensayos controlados aleatorios, en la que primero se identifica la pregunta de interés, que luego determina las herramientas y los procedimientos de medición específicos seleccionados para el propósito definido. Posterior a ello, en el proceso de investigación basada en la práctica en psicoterapia, se identifica el problema de interés para los profesionales o administradores del servicio, el cual se aborda con los datos recopilados en la práctica rutinaria; esta etapa sería equivalente al análisis de resultados de un experimento o ensayo controlado aleatorio estándar. Es importante destacar que los análisis y procedimientos empleados probablemente tengan un alcance más amplio, en términos de usabilidad, que los utilizados en los ensayos controlados aleatorios tradicionales. Finalmente, los resultados contribuyen al desarrollo de un ciclo de retroalimentación, es decir, que la nueva información conduce a cambios en la práctica y a la recopilación de datos adicionales después de la implementación de los cambios.

Como cualquier otro paradigma, el de la EBP no está exento de amenazas a su validez. La primera de ellas se relaciona con la calidad de los datos, aspecto relacionado con la adecuada elección de las medidas de evaluación y de la completitud de los datos. El paradigma no se centra en la necesidad de recoger el 100\% de los datos, pero si en la recolección sistemática en muestras amplias de personas, por lo que se debe atender al número de casos incluidos por los profesionales, revisando que no se hagan procesos de selección de los mismos, y, además, se debe considerar la participación o no de todos los profesionales del servicio, teniendo en cuenta que quienes participen deben estar dispuestos a recolectar datos de forma sistemática. En segundo lugar, se encuentra el aspecto de la administración del tratamiento, a diferencia de los estudios de eficacia, los estudios basados en la práctica en psicoterapia se relacionan con el conocimiento sobre la naturaleza de las intervenciones que se están administrando, de allí que no se utilicen manuales de tratamiento ni se controle la implementación de una intervención particular. A cambio, deben considerarse políticas de recolección de datos sobre el cumplimiento del tratamiento (p.e. número de sesiones realizadas, desarrollo de las mismas y evolución del paciente), enfocándose en la calidad de los resultados dentro del sistema general de prestación de servicios, en lugar del funcionamiento de cualquier tipo específico de terapia psicológica (Barkham et al., 2010).

Un componente esencial en la aplicación del paradigma de la EBP en centros de atención en salud mental es el trabajo en torno al clima organizacional, ya que un adecuado ambiente contribuye a la maximización en la recolección y el uso de los datos; es así como la aplicación de este paradigma también puede asumirse como una intervención organizacional. En este sentido, la adopción de un sistema unificado de evaluación de resultados beneficia tanto al profesional como al gerente del servicio, a la vez que contribuye al trabajo sobre el liderazgo organizacional (Cape \& Barkham, 2002).

Definitivamente, la implementación del paradigma de la EBP en contextos de atención en salud mental requiere de planificación y esfuerzo, sin embargo, los beneficios de su aplicación pueden ser amplios en razón de promover la generación de evidencia propia y ajustada a las necesidades de los servicios, a un menor costo en comparación con los estudios de eficacia. Este paradigma puede ser una solución para la generación de evidencia 
Pág 7

Para obtener los beneficios del paradigma de la Evidencia Basada en la Práctica en los sistemas de atención en salud mental, se requiere de un compromiso con la filosofía planteada por el paradigma, acompañado de una infraestructura adecuada para el apoyo de las actividades de investigación y la capacidad técnica suficiente para recolectar y compartir datos. en psicoterapia en Latinoamérica, región donde no siempre se tienen los recursos para desarrollar estudios de eficacia, y en la que se requiere de información sobre la efectividad de los tratamientos con el fin de optimizar los recursos existentes.

\section{Sistema de salud en Latinoamérica, la necesidad de evidencia}

En las últimas décadas la práctica profesional en psicoterapia se ha extendido en los diferentes países de Latinoamérica. Uno de los motivos de esta expansión se debe a que en los últimos 30 años los gobiernos de Latinoamérica han procurado restructurar sus sistemas de salud mental en función de adaptarse a las propuestas indicadas en la Declaración de Caracas (Panamerican Health Organization \& World Health Organization, 1990). Estas propuestas pretenden movilizar la atención de la salud mental de un modelo basado en el internamiento en hospitales psiquiátricos a un modelo basado en la atención comunitaria. En consecuencia, los gobiernos de diferentes países de la región han planteado reformas y planes de salud mental en los que se determina la creación de centros de salud mental comunitarios, programas de atención ambulatoria y el entrenamiento de profesionales para la atención primaria en salud mental (Caldas-de-Almeida, 2013). A pesar de que, en muchos casos, estas políticas públicas de salud mental están visibilizadas, se desconoce si están siendo aplicadas, si se han implementado apropiadamente y si han servido para mejorar la salud mental de la población en las que se han efectuado (Alarcón, 2003; Henao et al., 2016).

Otro motivo por el que se ha extendido la práctica profesional de la psicoterapia en Latinoamérica obedece a que la práctica de la psicología clínica, como especialidad, ha aumentado en los últimos años en la región, tanto así, que es una de las primeras opciones de práctica profesional entre los estudiantes de psicología (Fernández-Alvarez \& Bregman, 2018). Por consiguiente, se ha incrementado la oferta de servicios de atención psicológica tanto a nivel público como privado; sin embargo, todavía existen limitaciones asociadas, como la falta de regulaciones a la práctica profesional y la disparidad en la calidad del entrenamiento en psicoterapia (Sanchez-Sosa, 2007). A esto, se suman las dificultades para crear nuevo conocimiento y la tendencia a aplicar programas de atención y tratamientos generados en países desarrollados sin adaptarlos a las condiciones sociales de la población en la que serán aplicados (Gutiérrez \& Landeira-Fernández, 2018) y sin evaluar su efectividad.

De hecho, la investigación en psicoterapia, aunque ha aumentado en los últimos años en la región, todavía está concentrada mayoritariamente en los países del cono sur; los cuales presentan mayor productividad e interés por esta área (De la Parra, 2013). Es evidente la escasez de datos sobre la efectividad de los tratamientos psicológicos aplicados en la región en diferentes niveles de atención, sean estos públicos o privados, y por tanto sobre la validez de los sistemas de atención en salud mental. En este contexto, el paradigma de la EBP en psicoterapia adquiere relevancia para generar información que pueda poner a prueba la efectividad de los tratamientos, técnicas e intervenciones utilizadas en la práctica habitual.

Para obtener los beneficios del paradigma de la EBP en los sistemas de atención en salud mental, se requiere de un compromiso con la filosofía planteada por el paradigma, acompañado de una infraestructura adecuada para el apoyo de las actividades de investigación y la capacidad técnica suficiente para recolectar y compartir datos. En el caso latinoamericano aún es difícil reconocer este modelo en el que los profesionales se apropian de la recolección de datos y los sistemas de medición rutinaria, generando utilidades a nivel individual y local, en sus propios centros y entre diferentes centros, alimentando un sistema de evidencia de orden superior sobre la efectividad de los tratamientos psicológicos 
La generación de estudios basados en el paradigma de la Evidencia Basada en la Práctica requiere de la recolección continua de datos dentro de la práctica clínica habitual de la psicoterapia. Estos datos deben ser lo suficientemente robustos para permitir la evaluación de resultados, por lo tanto, la recolección de datos a gran escala es de vital importancia. Para cumplir con este requerimiento, es fundamental el desarrollo y creación de Redes de Investigación en la Práctica (RIP; denominadas en inglés como Practice Research Network). aplicados (Fernández-Alvarez \& Castonguay, 2018). Es posible que en el contexto regional las distancias entre la práctica y la investigación sean muy variadas y la cultura de recolección de datos en algunos países sea aún incipiente; sin mencionar la pluralidad en la formación de psicólogos creadores, sintetizadores y consumidores de evidencia proveniente de la investigación, que sean capaces de incorporar las habilidades para la recolección y utilización de datos en su experiencia clínica, y para involucrar a sus pacientes en la toma de decisiones compartidas (Spring, 2007).

Bajo este panorama, el reto supone la construcción de una base común de conocimiento, investigación y práctica, que permita considerar y decidir sobre el tipo de información que se debe recopilar, estimulando en simultáneo a proveedores de tratamiento, terapeutas, pacientes e investigadores interesados (Fernández-Alvarez \& Castonguay, 2018). Todo ello, sin perder de vista la variabilidad propia de esta región, que incluye países con mayor trayectoria que a partir de su experiencia pueden apoyar a los países que hasta ahora comienzan a contribuir en el desarrollo del campo de la investigación en psicoterapia.

\section{Redes de Investigación en la Práctica}

La generación de estudios basados en el paradigma de la EBP requiere de la recolección continua de datos dentro de la práctica clínica habitual de la psicoterapia. Estos datos deben ser lo suficientemente robustos para permitir la evaluación de resultados, por lo tanto, la recolección de datos a gran escala es de vital importancia. Para cumplir con este requerimiento, es fundamental el desarrollo y creación de Redes de Investigación en la Práctica (RIP; denominadas en inglés como Practice Research Network). Las RIP nacen a partir de la necesidad de crear vías de comunicación para trasladar las directrices clínicas a la práctica clínica habitual, es decir, la creación de un vínculo entre investigadores y terapeutas (Lucock et al., 2017). Las RIP tienen sus orígenes en el campo de la atención primaria en medicina a partir de la sistematización de los datos recolectados por varios clínicos acerca de la evolución de sus pacientes (Barkham, 2014); y de grupos aislados de clínicos que se han convertido en redes de colaboración a nivel de país, como los casos de Estados Unidos y Reino Unido (Parry, Castonguay, Borkovec, \& Wolf, 2010).

Las RIP consisten en un conjunto de clínicos que colaboran para conducir procesos de investigación (Zarin, Pincus, West, \& Mcintyre, 1997), que responden a preguntas significativas que surgen en contextos naturalísticos y de esta manera se atiende a la validez externa; mientras se aplica una metodología rigurosa para no dejar de lado la validez interna (Borkovec, 2002). Por tanto, las RIP en psicoterapia tienen como fin desarrollar una infraestructura colaborativa de profesionales con un mismo objetivo, investigar las cuestiones habituales de la práctica clínica cotidiana, es decir, los problemas que se presentan cuando se aplican intervenciones psicológicas en ambientes poco controlados. Como ejemplo de este tipo de asociaciones, Farfallini et al. (2018) describen el proceso de construcción de una Red de Investigación Orientada por la Práctica y presentan los resultados de dos estudios sobre actitudes y toma de decisiones en terapeutas de España y Argentina. La formación de una RIP supone la creación de una estructura compuesta por varios elementos: una batería común de medidas de evaluación, procedimientos de evaluación estandarizados y un marco común para la evaluación de proyectos de investigación (Castonguay, Pincus, \& McAleavey, 2015). Según Parry et al. (2010), el componente primordial en la creación de una RIP es acordar un sistema común para la recolección de resultados y apreciar los cambios en psicoterapia, lo que se logra a través del uso de una medida o conjunto de medidas comunes en todos los servicios involucrados. Esto permite desarrollar bases de datos con muestras amplias y clínicamente representativas, las cuales pueden ayudar a responder preguntas de investigación complejas y que se adhieran a los vacíos 
de conocimiento sobre la práctica habitual de la psicoterapia. Las medidas de cambio y resultado en psicoterapia en este contexto deben permitir la monitorización rutinaria del progreso en la psicoterapia.

Existen diversas medidas desarrolladas con el objetivo de medir el cambio de manera rutinaria. Drapeau (2012) identificó 10 medidas que cumplen este propósito y son utilizadas en diferentes países alrededor del mundo. A nivel regional, Paz, Mascialino, Proaño y Evans (2020) revisaron la utilización de medidas de resultado en Latinoamérica e identificaron el Outcome Questionnaire, el Beck Depression Inventory-II, el Hamilton Rating Scale y el Yale-Brown Obsessive-Compulsive Scale como las escalas de mayor frecuencia. Entre las medidas específicas de resultado en psicoterapia, se destacan dos que han ido fortaleciendo su trayectoria en el campo de la investigación en psicoterapia en Latinoamérica: Outcome Questionnaire 45.2 (OQ-45.2; Lambert et al., 1996) y Clinical Outcomes in Routine Evaluation-Outcome Measure (CORE-OM; Evans et al., 2002, 2000).

El OQ-45.23 (Lambert et al., 1996) es un cuestionario de autoreporte diseñado para monitorizar los resultados del tratamiento en contextos de salud mental. Contiene 45 ítems divididos en tres subescalas: Symptom Distress (SD) para medir los síntomas en general, Interpersonal Relations (IR) para valorar las relaciones interpersonales y Social Role (SR) para evaluar el rol social del individuo. Los ítems son evaluados en escala tipo Likert de cinco puntos (0-4). La versión original en inglés de este cuestionario es ampliamente utilizada, especialmente en Estados Unidos (Boswell, Kraus, Miller, \& Lambert, 2015) y demostró tener propiedades psicométricas aceptables (Lambert et al., 1996). En Latinoamérica el cuestionario fue traducido al español en Chile (De La Parra, Von Bergen, \& Del Río, 2002) y presentó propiedades psicométricas similares a la versión original. Una versión más corta del cuestionario fue desarrollada en Chile, el OQ-30.2 (Errázuriz, Opazo, Behn, Silva, \& Gloger, 2017), y presentó propiedades psicométricas similares a la versión de 45 ítems. En Colombia, Londoño et al., (2017) publicaron resultados sobre la validación del OQ 45.2 en una muestra clínica, sus resultados mostraron una adecuada sensibilidad al cambio, sin embargo, el cuestionario no presentó una estructura adecuada a través del análisis factorial confirmatorio.

El CORE-OM ${ }^{4}$ (Evans et al., 2002, 2000) es una medida auto reportada conformada por 34 ítems que evalúan cuatro dominios: Bienestar psicológico, Problemas/Síntomas, Funcionamiento y Riesgo. Los ítems son puntuados en escala Likert de 0 (nunca) a 4 (siempre o casi siempre). Los puntajes más altos indican niveles de mayor malestar psicológico. La versión original del cuestionario, en inglés, presenta buenas propiedades psicométricas (Evans et al., 2002) y es utilizada principalmente en países europeos (Boswell et al., 2015). El CORE-OM fue traducido al español en España y presenta propiedades psicométricas similares a la versión original (Trujillo et al., 2016). Una exploración psicométrica de las propiedades del CORE-OM en población no clínica de Ecuador (Paz, Mascialino, \& Evans, 2020) evidenció que son comparables a las encontradas en los estudios de Evans et al. (2002) con la versión original y Trujillo et al. (2016) con la traducción al español. En la actualidad existen esfuerzos en varios países de Latinoamérica por comprobar las propiedades psicométricas de la versión en español de este cuestionario, algunos de los avances parciales de estos estudios de validación fueron presentados en la 50ma reunión internacional anual de la Sociedad de Investigación en Psicoterapia, en la que países como Ecuador (Paz, Evans, \& Mascialino), Colombia (Ortiz, Trujillo, \& Domínguez), Chile (Errazuriz, Gómez-Barris, Franziska, \& De la Parra), Uruguay (Dogmanas, Arias, Álvarez-Vaz, \& Vitale) y Brasil (Fukuda, Cascard, \& Fleury) expusieron y discutieron sus resultados (Paz, 2019).

3. El uso del $O Q-45.2$ y sus variantes está restringido por una licencia que puede ser adquirida en: http://www.oqmeasures.com 
El paradigma de la Evidencia Basada en la Práctica puede ampliar los horizontes de conocimiento sobre psicoterapia y atención en salud mental en Latinoamérica.

Por sus características, este paradigma puede mostrar y consolidar los conocimientos existentes actualmente en la práctica habitual de psicoterapia en la región latinoamericana a través de la sistematización de esta información.
El uso consistente y amplificado de estas medidas podría promover la creación de RIP en el ámbito de las intervenciones psicológicas en Latinoamérica, con investigadores y clínicos que usen sistemas comunes de evaluación y por lo tanto puedan disponer de un lenguaje similar para entender la evidencia recolectada. Esto permitiría la creación de evidencia desde las realidades propias de los psicoterapeutas involucrados de cada uno de los países, así como de la región en conjunto. Por tanto, además de la validación de las medidas de resultado, es relevante su difusión en el ámbito de la práctica, para lo cual es necesario el compromiso de los investigadores y de las redes emergentes para ofrecer información sobre las medidas de cambio y resultado ya validadas, y sobre los beneficios de su utilización en el contexto clínico. Cuando se trata de la utilización de estas medidas, algunas de las recomendaciones expuestas en el estudio realizado por Paz et al. (2020) podrían ser consideradas: en primer lugar, promover la utilización de medidas de resultado y cambio, ya que estas pueden adaptarse adecuadamente a la realidad diversa y cambiante de los países latinoamericanos; y, en segundo lugar, plantear la conveniencia de usar medidas generales de cambio, en lugar de medidas dirigidas a síntomas específicos.

La creación de RIP exige compromiso y cooperación por parte de los involucrados, los objetivos deben ser claros y acordados en conjunto; pero, sobre todo, la red debe tener como fin la difusión de resultados de manera abierta y transparente. Las desigualdades económicas y sociales son evidentes dentro de cada uno de los países y entre los diferentes países de la región, por eso, promover la creación de evidencia cooperativa, ofreciendo medidas de resultado gratuitas y con resultados de acceso libre a través de las RIP, permitirá mayor acceso al conocimiento sobre la práctica clínica y la investigación en psicoterapia en Latinoamérica.

\section{Conclusiones}

El paradigma de la EBP puede ampliar los horizontes de conocimiento sobre psicoterapia y atención en salud mental en Latinoamérica. Por sus características, este paradigma puede mostrar y consolidar los conocimientos existentes actualmente en la práctica habitual de psicoterapia en la región latinoamericana a través de la sistematización de esta información. Para comenzar a generar estudios y prácticas basados en el paradigma de la EBP es necesaria la creación de RIP, las cuales se definen como una infraestructura colaborativa de profesionales con objetivos concretos y compartidos de investigación. El primer paso para conformar las RIP es definir los objetivos de investigación y elegir una medida de monitorización de resultados común. La elección de la medida debe estar regida por la accesibilidad a la misma y la capacidad de los miembros de la red para utilizarla. En el escenario latinoamericano, el CORE-OM podría cumplir con tales requerimientos, al ser una medida gratuita y que puede ser utilizada en diferentes tipos de consultantes y con diferentes tipos de enfoques de tratamiento psicológico. Así mismo, la red debe ser capaz de generar un sistema de recolección y procesamiento de datos que se ajuste a las necesidades de los miembros y a los objetivos de investigación propuestos. En la 50ma reunión internacional anual de la sociedad de Investigación en Psicoterapia se propuso la creación de una red de investigación en la práctica para la región, considerando como eje fundamental la evaluación de resultados. La idea fue acogida por investigadores de diferentes países (Ecuador, Chile, Colombia, Brasil y Uruguay) y se espera que pueda consolidarse en los próximos años a través de la ya creada Red Latinoamericana de Investigación en Psicoterapia y Cambio $^{5}$. 


\section{Referencias}

Alarcón, R. D. (2003). Mental health and mental health care in Latin America. World Psychiatry: Official Journal of the World Psychiatric Association (WPA), 2(1), 54546.

APA Presidential Task Force on Evidence-Based Practice. (2006). Evidence-based practice in psychology. The American Psychologist, 61(4), 271.

Barkham, M. (2014). Practice-based research networks: Origins, overview, obstacles, and opportunities. Counselling and Psychotherapy Research, 14(3), 167-173. https://doi.org/10.1080/14733145.2014.929414

Barkham, M., Delgadillo, J. Firth, N. \& Saxon, D. (2018). Practice based evidence and the law of variability in psychological treatment. Revista Argentina de Clínica Psicológica, 27(12), 115-135. https://doi.org/10.24205/03276716.2018.1059

Barkham, M., \& Margison, F. (2007). Practice-based evidence as a complement to evidence-based practice: From dichotomy to chiasmus. En C. Freeman \& M. Power (Eds), Handbook of evidence-based psychotherapies: A guide for research and practice, (pp. 443-476). Londres, Inglaterra: John Wiley \& Sons Ltd.

Barkham, M., \& Mellor-Clark, J. (2000). Rigour and relevance: practice-based evidence in the psychological therapies. En N. Rowland, \& S. Goss (Eds), Evidence based counselling and psychological therapies (pp. 127-144). Londres, Inglaterra: Routledge.

Barkham, M., \& Mellor-Clark, J. (2003). Bridging evidence-based practice and practice-based evidence: Developing a rigorous and relevant knowledge for the psychological therapies. Clinical Psychology \& Psychotherapy, 10(6), 319-327.

Barkham, M., Stiles, W. B., Lambert, M. J., \& Mellor-Clark, J. (2010). Building a rigorous and relevant knowledge-base for the psychological therapies. En M. Barkham, G. E. Hardy, \& J. Mellor-Clark (Eds). Developing and delivering practice-based evidence: A guide for the psychological therapies, (pp. 329-353). Londres, Inglaterra: John Wiley \& Sons Ltd.

Borkovec, T. D. (2002). Training clinic research and the possibility of a national training clinics practice research network. The Behavior Therapist, 25, 98-103.

Boswell, J. F., Kraus, D. R., Miller, S. D., \& Lambert, M. J. (2015). Implementing routine outcome monitoring in clinical practice: Benefits, challenges, and solutions. Psychotherapy Research, 25(1), 6-19. https://doi.org/10.1080/10503307.2013.8 17696

Caldas-de-Almeida, J. M. (2013). Mental Health Care Reforms in Latin America: An Overview of Mental Health Care Reforms in Latin America and the Caribbean. International Health, 5(1), 15-18. https://doi.org/10.1093/inthealth/ihs013

Cape, J., \& Barkham, M. (2002). Practice improvement methods: Conceptual base, evidence-based research, and practice-based recommendations. British Journal of Clinical Psychology, 41(3), 285-307. https://doi. org/10.1348/014466502760379145

Castonguay, L. G., Pincus, A. L., \& McAleavey, A. A. (2015). Practice research network in a psychology training clinic: Building an infrastructure to foster early attachment to the scientific-practitioner model. Psychotherapy Research, 25(1), 52-66. https://doi.org/10.1080/10503307.2013.856045

De la Parra, G. (2013). Psychotherapy research in developing countries: The case of Latin America. Psychotherapy Research, 23(6), 609-623. https://doi.org/10.1080 110503307.2013 .830794

De La Parra, G., Von Bergen, A., \& Del Río, M. (2002). Primeros hallazgos de la aplicación de un instrumento que mide resultados psicoterapéuticos en una muestra de pacientes y de población general. Revista Chilena de Neuro-Psiquiatria, 40(3), 201-209. https://doi.org/10.4067/S0717-92272002000300003 
Drapeau, M. (2012). The value of tracking in psychotherapy. Integrating Science and Technology, 2(2). https://doi.org/10.1.1.680.46.34

Errázuriz, P., Opazo, S., Behn, A., Silva, O., \& Gloger, S. (2017). Spanish Adaptation and Validation of the Outcome Questionnaire OQ-30.2. Frontiers in Psychology, 8(673), 1-9. https://doi.org/10.3389/fpsyg.2017.00673

Evans, C., Connell, J., Barkham, M., Margison, F., McGrath, G., Mellor-Clark, J., \& Audin, K. (2002). Towards a standardised brief outcome measure: Psychometric properties and utility of the CORE-OM. British Journal of Psychiatry, 180(01), 51-60. https://doi.org/10.1192/bjp.180.1.51

Evans, C., Mellor-Clark, J., Margison, F., Barkham, M., Audin, K., Connell, J., \& McGrath, G. (2000). CORE: Clinical Outcomes in Routine Evaluation. Journal of Mental Health, 9(3), 247-255.

Farfallini. L., Molinari, G., Espinoza, M., Suso Ribera, C., Gómez Penedo, J. M., \& Fernández-Álvarez, J. (2018). Building a practice research network of young therapists and early career researchers in spanish speaking countries: why, who \& how. Revista Argentina de Clínica Psicológica, 27(2), 203-228. https://doi. org/10.24205/03276716.2018.1067

Fernández-Alvarez, H., \& Bregman, C. (2018). Clinical Psychology and Health Psychology. En R. Ardila (Ed) Psychology in Latin America: Current Status, Challenges and Perspectives, (pp. 53-75). Bogotá, Colombia: Springer. https://doi. org/10.1007/978-3-319-93569-0 4

Fernández-Alvarez, H., \& Castonguay, L. G. (2018). Practice-Oriented Research: Advances in Clinicians-researchers collaborations. Introduction. Revista Argentina de ClínicaPsicológica,27(2),107-114.https://doi.org/10.24205/03276716.2018.1069

Gérvas, J., \& Pérez A, C. (2008). Evaluación de las intervenciones en salud: la búsqueda del equilibrio entre la validez interna de los resultados y la validez externa de las conclusiones. Revista Española de Salud Pública, 82, 577-579.

Green, D., \& Latchford, G. (2012). Maximising the benefits of psychotherapy: A practice-based evidence approach. Oxford, United Kingdom: John Wiley \& Sons.

Gutiérrez, G., \& Landeira-Fernández, J. (2018). Psychological Research in Latin America: Current and Future Perspectives. En R. Ardila (Ed) Psychology in Latin America: Current Status, Challenges and Perspectives (pp. 7-26). https://doi. org/10.1007/978-3-319-93569-0 2

Henao, S., Quintero, S., Echeverri, J., Hernández, J., Rivera, E., \& López, S. (2016). Políticas públicas vigentes de salud mental en Suramérica: un estado del arte. Revista Facultad Nacional de Salud Pública, 34(2). https://doi.org/10.17533/udea. rfnsp.v34n2a07

Holmqvist, R., Philips, B., \& Barkham, M. (2015). Developing practice-based evidence: Benefits, challenges, and tensions. Psychotherapy Research, 25(1), 20-31. https:// doi.org/10.1080/10503307.2013.861093

Jacobson, N.S., and Christensen, A. (1996). Studying the effectiveness of Psychotherapy. How well can clinical trials do the job? American Psychologist, 51(10), 1031-1039.

Kazarian, S. S., \& Evans, D. R. (2001). Health psychology and culture: Embracing the 21 st century. En S. S. Kazarian \& D. R. Evans (Eds.), Handbook of Cultural Psychology (pp. 837-846). San Diego, CA, US: Academic Press.

Kazdin, A. E. (2014). Evidence-based psychotherapies I: Qualifiers and limitations in what we know. South African Journal of Psychology, 44(4), 381-403.

Lambert, M. J., Hansen, N., Umphress, V., Lunnen, K., Okiishi, J., Burlingamen, G., \& Reisinger, C. (1996). Administration and scoring manual for the OQ-45.2. Wilmington, DE: American Professional Credentialing Services. 
Lilienfeld, S. O., Ritschel, L. A., Lynn, S. J., Cautin, R. L., \& Latzman, R. D. (2013). Why many clinical psychologists are resistant to evidence-based practice: Root causes and constructive remedies. Clinical psychology review, 33(7), 883-900.

Lucock, M., Barkham, M., Donohoe, G., Kellett, S., McMillan, D., Mullaney, S., ... Delgadillo, J. (2017). The Role of Practice Research Networks (PRN) in the Development and Implementation of Evidence: The Northern Improving Access to Psychological Therapies PRN Case Study. Administration and Policy in Mental Health and Mental Health Services Research, 44(6), 919-931. https://doi.org/10.1007/ s10488-017-0810-5

Londoño, N. H., Agudelo, D. M., Martínez, E., Anguila, D., Aguirre, D. C., \& Mogollón, C. (2017). Cuestionario de efectividad de la psicoterapia Outcome Questionnaire. Validación en muestra clínica colombiana. Psychologia. Avances de la disciplina, 11(1), 97-108.

Margison, F., Barkham, M., Evans, C., McGrath, G., Mellor-Clark, J., Audin, K., \& Connell, J. (2000). Measurement and psychotherapy: evidence-based practice and practice-based evidence. British Journal of Psychiatry, 177, 123-130.

Martínez-Taboas, A. (2014). Prácticas psicológicas basadas en la evidencia: beneficios y retos para Latinoamérica. Revista Costarricense de Psicología, 33(2), 63-78.

McMorrow, E., \& Carr, A. (2003). How do we find out what works for whom? Evaluating the efficacy and effectiveness of psychotherapy. Eisteacht, 2(26), 24-31.

Nathan, P. E. (2007). Efficacy, effectiveness, and the clinical utility of psychotherapy research. The art and science of psychotherapy, 69-83.

Nathan, P.E., Stuart, S.P., and Dolan, S.L. (2000). Research on psychotherapy efficacy and effectiveness: Between Scylla and Charybdis? Psychological Bulletin, 126(6), 964-981.

National Advisory Mental Health Council [NAMHC]. (1999). Bridging science and service: A report by the National Advisory Mental Health Council's Clinical treatment and Services Research Workshop (NIH Publication No. 99-4353). Washington DC.

O'Donohue, W., \& Ferguson, K. E. (2006). Evidence-based practice in psychology and behavior analysis. The Behavior Analyst Today, 7(3), 335-350. https://doi. org/10.1037/h0100155

Panamerican Health Organization, \& World Health Organization. (1990). The Caracas Declaration. Conference on Restructuring Psychiatric Care in Latin America within the Local Health Systems. Recuperado de http://www.globalhealthrights.org/ wp-content/uploads/2013/10/Caracas-Declaration.pdf\%0Ahttp://www2.paho. org/hq/dmdocuments/2008/DECLARATIONOFCARACAS.pdf

Parry, G., Castonguay, L. G., Borkovec, T. D., \& Wolf, A. W. (2010). Practice Research Networks and Psychological Services Research in the UK and USA. En M. Barkham, G. E. Hardy, \& J. Mellor-Clark (Eds.), Developing and Delivering Practice-Based Evidence: A guide for the psychological therapies (pp. 311-325). Londres, Inglaterra: John Wiley \& Sons Ltd.

Paz, C. (2019) Findings of the use of Routine Outcome Measures in Latin America. Panel presentado en la 50th Annual International Meeting de la Society for Psychotherapy Research, Buenos Aires, Argentina.

Paz, C., Mascialino, G., \& Evans, C. (2020). Exploration of the psychometric properties of the Clinical Outcomes in Routine Evaluation-Outcome Measure in Ecuador. BMC Psychology, 8(1), 94. https://doi.org/10.1186/s40359-020-00443-z

Paz, C., Mascialino, G., Proaño, K., \& Evans, C. (2020). Psychological intervention change measurement in Latin America: Where from? Where to?. Psychotherapy Research, 1-10. https://doi.org/10.1080/10503307.2020.1750728 
Preciado, J. (2012). Culture in Ibero-America: A neglected issue in behavioral and cognitive randomized control trial interventions. International Journal of Clinical and Health Psychology, 12(3), 489-501.

Resnicow, K., Soler, R., Braithwaite, R. L., Ahluwalia, J. S. \& Butler, J. (2000). Cultural sensitivity in substance use prevention. Journal of Community Psychology, 28(3), 271-290.

Sanchez-Sosa, J. J. (2007). Psychotherapy in Mexico: Practice, training, and regulation. Journal of Clinical Psychology, 63(8), 765-771. https://doi.org/10.1002/ jclp.20391

Shadish, W. R., Matt, G. E., Navarro, A. M., Siegle, G., Crits-Christoph, P., Hazelrigg, M. D., ... Weiss, B. (1997). Evidence that therapy works in clincally representative conditions. Journal of Consulting and Clinical Psychology, 65(3), 355-365. https:// doi.org/10.1037/0022-006X.65.3.355

Spring, B. (2007). Evidence-based practice in clinical psychology: What it is, why it matters; what you need to know. Journal of Clinical Psychology, 63(7), 611-631. https://doi.org/10.1002/jclp.20373

Trujillo, A., Feixas, G., Bados, A., Garcia-Grau, E., Salla, M., Medina, J. C., ... Evans, C. (2016). Psychometric properties of the spanish version of the clinical Outcomes in routine evaluation-Outcome Measure. Neuropsychiatric Disease and Treatment, 12, 1457. https://doi.org/10.2147/NDT.S103079

White, J. W., Koss, M. P., \& Kazdin, A. E. (Eds.). (2011). Violence against women and children (Vols. 1-2). Washington, DC: American Psychological Association.

Zane, N., Hall, G. C. N., Sue, S., Young, K., \& Núñez, J. (2004). Research on psychotherapy with culturally diverse populations. En M. J. Lambert (Ed.), Bergin and Garfield's Handbook of Psychotherapy and Behavior Change (pp. 767-804). New York, US: Wiley

Zarin, D. A., Pincus, H. A., West, J. C., \& Mcintyre, J. S. (1997). Practice-Based Research in Psychiatry. American Journal of Psychiatry, 154(9), 1199-1208. 